\title{
The Critical Point and particle correlations under thermal stochastic influence
}

\author{
Gennady Kozlov*i \\ JINR \\ E-mail: kozlovejinr.ru
}

The phase transition of baryonic matter is studied in the model of random fluctuation walk. The solution contains the regular and the singular non-analytical parts for baryonic matter (BM) and anomaly matter (AM), respectively. The stochastic fields influence intrinsic to Bose-Einstein correlations of identical particles is a particular ingridient of the model. The conditions to phase transition from BM to AM are found.

Critical Point and Onset of Deconfinement - CPOD2017

7-11 August, 2017

The Wang Center, Stony Brook University, Stony Brook, NY

* Speaker.

${ }^{\dagger}$ A footnote may follow. 


\section{Introduction}

The critical behavior of baryonic matter (BM) and its transition to anomaly matter (AM), containing free fields of quarks and gluons at high temperature $T$ and baryon density, has a great interest, in particular, with scanning over QCD variables to predict the Critical Point (CP). The latter is characterized by the critical temperature $T_{c}$ and the critical baryon chemical potential $\mu_{c}$. The large enough baryon density may be a source of spontaneous breaking of space parity of colliding particles (see, e.g., [1]). This breaking can be manifested in the form of chiral magnetic effect [2] at non-central collisions of heavy ions. In addition, the local breaking of space parity (LBSP) of colliding nuclei may lead to anomaly yield of leptonic pairs [3,4]. LBSP may arise also in case of Bose-Einstein condensation (BEC) of light pseudoscalar particles, e.g., pions. The appearance of light hadron or even heavier flavor condensates in hot and high density nuclear matter has not been discovered yet, and this might be one of the goals to the search programs of heavy ion colliders and accelerators with fixed targets operating at high enough baryon density.

It is known that the selfconsistent description of the critical behavior of a dynamical system in field theory is given by the renormalization group (RG) (see, e.g., [5-7] and the refs. therein). In $\mathrm{RG}$ approach to field theory the particular case of the phase transition is defined by the presence and the properties of the fixed points. As an example, the infra-red (IR) attracted fixed point is relevant to the phase transition of the second kind as well as to the critical scaling. However, the fluxes (solutions) of RG may leave the physical domain containing the IR fixed point (IRFP). The solutions may even go to infinity. Such a situtaion can be considered in terms of the phase transition of the first kind. In the neighborhood of an IRFP the strong coupling constant is

$$
\alpha^{I R F P} \simeq-2 \pi \frac{b_{0}}{b_{1}}, b_{0}=\frac{1}{3}\left(11 N_{c}-2 N_{f}\right),
$$

where $N_{c}$ and $N_{f}$ are numbers of colors and flavors, respectively; $b_{0}$ and $b_{1}$ are the coefficients in the $\beta$ - function

$$
\beta(\alpha) \equiv \bar{\mu} \frac{\partial \alpha}{\partial \bar{\mu}}=-\frac{b_{0}}{2 \pi} \alpha^{2}-\frac{b_{1}}{(2 \pi)^{2}} \alpha^{3}+\ldots
$$

at some scale $\bar{\mu}$ for $S U\left(N_{c}\right)$ gauge theory. The scale of chiral-symmetry restoration and the associated deconfinement scale are of the order $\Lambda$ at which $\alpha(\bar{\mu})$ crosses the critical value $\alpha_{c}$. If $N_{f}$ decreases ( $\alpha^{I R F P}$ increases) CP is characterized by $\alpha_{c}<\alpha^{I R F P}$ at which the spontaneous breaking of chiral symmetry is occurred and confinement does appear. Actually, the phase transition does influence the particle source size.

\section{Size of a particle source}

One of useful and instructive approaches to study $\mathrm{CP}$ is through the spatial correlations of the final state particles. To observe the effective size of the source of particles produced in, e.g., heavy ion collisions, one needs to derive the theoretical formulas for two- or multi-particle distributioncorrelation functions and the function of chaoticity of particles. The very popular hydrodynamical models can only describe the one-particle distribution, but never give any kind of particle number fluctuations and multi-particle correlations. We have already studied this subject in the frame 
of searching CP through the correlations between identical particles with Bose statistics, BoseEinstein correlations (CBE), where the main object is the stochastic scale $L_{s t}$ which defines the effective evolving size of the source of particles in hot excited matter [8]. $L_{s t}$ depends on the temperature in the system (bath), the transverse momentum of two correlated particles, and it feels the influence of random stochastic fields parametrised by the function of chaoticity strength $v$ which goes to zero when $\mathrm{CP}$ is approached. $L_{s t}$ enters the $\mathrm{CBE}$ function $C_{2}(q ; \lambda)$ in a simple form (for details, see [9])

$$
C_{2}(q ; \lambda) \simeq \eta(N)\left[1+\lambda(v) e^{-q^{2} L_{s t}^{2}}\right](1+\delta \cdot q+\ldots),
$$

where $\eta(N)=\langle N(N-1)\rangle /\langle N\rangle^{2}$ gives "event-to-event" fluctuations to particle multiplicity $N$; $q$ is the relative momentum between two particles; $\lambda(v) \simeq 1 /(1+v)^{2}$, where $0<v<\infty$ [10]; $\delta$ is the measure of long-range correlation effect. Actually, $\delta \rightarrow 0$ at large enough space separation between two correlated particles. On the other hand, in [11] there was reported an anti-correlation effect in which the measured function $C_{2}$ attained values below the asymptotic minimum at unity, similarly to what had previously been observed in $e^{+} e^{-}$collisions [12]. Phenomenological aspects of this dip-effect have been noted in [13].

The scaling form (2.1) is useful to predict the behavior of observables as well as to indicate the vicinity of CP. Note, that $L_{s t} \rightarrow \infty$ as $T \rightarrow T_{c}$ and $\mu \rightarrow \mu_{c}$. The observables may be associated, e.g., with the transverse momentum $k_{T}=\left|\vec{p}_{T_{1}}+\vec{p}_{T_{2}}\right|$ of two Bose particles with momenta $\left|\vec{p}_{T_{i}}\right|=$ $\sqrt{\vec{p}_{x_{i}}^{2}+\vec{p}_{y_{i}}^{2}}, i=1,2$ at high enough $T[9]$ :

$$
k_{T}^{2}=\frac{1}{v(n) T^{3} L_{s t}^{5}}, n(\beta)=\frac{1}{e^{(\omega-\mu) \beta-1}}, \quad p_{\mu}=(\omega, \vec{p}) .
$$

The results of $e^{+} e^{-}$collisions at LEP [14] as well as ones at the LHC energies by CMS [11,15], ALICE [16] and ATLAS [17] experiments were indicated to decreasing of a size of a particle source with increasing $k_{T}$ which is supported by (2.2) at finite $T$. The coherence function $0<\lambda<1$ in (2.1) has been measured in many experiments (see, e.g., the refs in [18]. However, it is more important to study $\lambda$ theoretically as a function of particle occupation number $n$ at the state with finite $T$ through the transition from a fully coherent phase $(\lambda \simeq 0)$ to the chaotic one $(\lambda \simeq 1)$, where the critical behavior in the phase transition between BM and AM can occur. Thus, one can study the occurrence of BEC in thermal bath and two-particle momentum correlation as a function of an energy, (mean) particle multiplicity and the temperature of the system, distorted by the external fields (the influence of an environement). BEC is a consequence of a hadronization process due to phase transition from free states of quarks and gluons (the vicinity of $\mathrm{CP}$ ) through the mixed phase state composed of hadrons, quarks and gluons. Sometimes, this is called as the cross-over walk. To search the phase transition from BM to AM we shall use the approach to random fluctuation walk with respect to chaoticity in correlations of identical particles.

\section{Random fluctuation walk}

We start with the model in which the phase transition between BM and AM modelling in 
one-dimensional $x$ - oriented axis approach is governed by the probability $P\left(x ; \bar{\lambda}, \mu_{c}\right)$

$$
P\left(x ; \bar{\lambda}, \mu_{c}\right)=p \sum_{j=0}^{\infty} \bar{\lambda}^{j} \frac{1}{2} \sqrt{\frac{\pi}{t}}\left[e^{-y_{-}^{2 j} / 4 t}+e^{-y_{+}^{2 j} / 4 t}\right],-\infty<x<\infty,
$$

where $\bar{\lambda}$ is the random fluctuation weigth, $y_{ \pm}^{j}=x \mu_{c} \pm a^{j}$, the running parameter $a=\left(\mu / \mu_{c}\right)>1$; $t=l \mu_{c}, l$ is the lattice spacing. In the limit $l \rightarrow 0\left(\mu_{c} \neq 0\right)$ one has (see also [19])

$$
P\left(x ; \bar{\lambda}, \mu_{c}\right)=p(\bar{\lambda}) \sum_{j=0}^{\infty} \bar{\lambda}^{j} \pi\left[\delta\left(x \mu_{c}-a^{j}\right)+\delta\left(x \mu_{c}+a^{j}\right)\right],
$$

where $p(\bar{\lambda})=(1-\bar{\lambda}) /(2 \pi)$ within the normalization condition $2\left(p+\bar{\lambda} p+\ldots+\bar{\lambda}^{j} p+\ldots\right)=1$, $0<\bar{\lambda} \leq 1$. The r.h.s. of (3.2) is the delta-shaped sequence of the r.h.s. (3.1) in the limit $t \rightarrow 0$. The limit $\bar{\lambda} \rightarrow 1$ in (3.2) admits the broad behavior of $P\left(x ; \bar{\lambda}, \mu_{c}\right)$ that means the vicinity of CP is approached. Contrary to that, $P\left(x ; \bar{\lambda}, \mu_{c}\right)$ will be trivial if $\bar{\lambda} \rightarrow 0: P(x ; \bar{\lambda} \rightarrow 0) \rightarrow 1 /(2 \pi)$.

In order to smooth the particularity of (3.2), one can use its Fourier transformed form $G\left(k ; \bar{\lambda}, \mu_{c}\right)$ which is nothing but the characteristic function. This provides the most convenient way to study an asymptotic behavior at large enough $x$ (or very sharp increasing of $L_{s t}$ in terms of CBE function (2.1)) corresponding to the limit $k \rightarrow 0$. The function $G\left(k ; \bar{\lambda}, \mu_{c}\right)$ has the form

$$
G\left(k ; \bar{\lambda}, \mu_{c}\right)=p(\bar{\lambda}) \sum_{j=0}^{\infty} \bar{\lambda}^{j} \cos \left(\frac{k}{\mu_{c}} a^{j}\right),
$$

accompanying by the properties: $2 \pi G(0 ; \bar{\lambda})=1, G^{\prime}(0 ; \bar{\lambda})=0$.

The fluctuation length $\xi$ is calculated through the even moments $M_{(2 s)}(\bar{\lambda})$ of the order $2 s$

$$
\xi_{(2 s)}^{2}(\bar{\lambda}) \sim M_{(2 s)}(\bar{\lambda})=\left.\frac{\partial^{2 s} G\left(k ; \bar{\lambda}, \mu_{c}\right)}{\partial k^{2 s}}\right|_{k=0} .
$$

Looking through the result

$$
\left|\xi_{(2 s)}^{2}(\bar{\lambda})\right|=p(\bar{\lambda}) \sum_{j=0}^{\infty}\left(\frac{a^{j}}{\mu_{c}}\right)^{2 s} \bar{\lambda}^{j}
$$

one can realize that (3.4) does converge at $\left(a / \mu_{c}\right)^{2 s} \bar{\lambda}<1$ only. In this case $\left|\xi^{2}\right|$ is finite

$$
\left|\xi_{(2 s)}^{2}(\bar{\lambda})\right| \simeq p(\bar{\lambda}) \mu_{c}^{-2 s}\left(1+a^{2 s} \bar{\lambda}\right) .
$$

However, if $\mu \simeq \mu_{c}$ and $\bar{\lambda}$ is close (but less) to 1 (this is a vicinity of CP), one finds the divergence of fluctuation length.

Actually, one can find an infinite number of divergent (singular) terms starting from (3.3), hence the latter does not suitable to describe an arbitrary phase of excited matter (wide values of $0<\bar{\lambda}<1$ and $\mu_{c}$, as well as small $k$ compared to $\mu_{c}$. To find the non-analytical part of (3.3) at $k=0$ let us start with functional scaling equation (see also [19])

$$
G\left(k ; \bar{\lambda}, \mu_{c}\right)=G_{B M}\left(k ; \bar{\lambda}, \mu_{c}\right)+G_{A M}\left(a k ; \bar{\lambda}, \mu_{c}\right),
$$

which is the linear non-homogenious equation, where

$$
G_{B M}\left(k ; \bar{\lambda}, \mu_{c}\right)=p(\bar{\lambda}) \cos \left(k / \mu_{c}\right),
$$




$$
G_{A M}\left(a k ; \bar{\lambda}, \mu_{c}\right)=\bar{\lambda} G\left(a k ; \bar{\lambda}, \mu_{c}\right) .
$$

(3.6) is the regular function at $k$ close to zero for all $0<\bar{\lambda}<1$ and it gives the contribution to BM. The phase with AM is provided by (3.7) at $a^{-2} \leq \bar{\lambda}<1$. The second term in (3.5) disappears if $\bar{\lambda} \rightarrow 0$, and the phase with BM does exist only. Here, we take into account that $G\left(a k ; \bar{\lambda} \simeq 0, \mu_{c}\right) \simeq$ $(1 / 2 \pi) \cos \left(k \mu / \mu_{c}^{2}\right)$ is finite. The same result at $\bar{\lambda} \simeq 0$ is expected if one uses (3.3).

Looking through (3.5) - (3.7) $\bar{\lambda}$ can be associated with the coherence function $\lambda(v)$, introduced in (2.1), where [8]

$$
v=\frac{1}{n k_{G L}^{2}} O\left(\frac{m_{\phi}^{2}}{m^{2}}\right) .
$$

Here, we have used the fact when CP is approaching, theory becomes conformal provided by the scalar field, the dilaton $\phi$, with the mass $m_{\phi}$. In (3.8), we use $m$ as a mass of (light) hadrons which are in the pattern of CBE; $k_{G L}=m_{\phi} / m_{B}$ is the Ginzburg-Landau parameter which can differ the vacuum of type-I $\left(k_{G L}<1\right)$ from those of type-II $\left(k_{G L}>1\right)$ in dual Higgs-Abelian gauge model with dual (to non-abelian gluon field $A_{\mu}^{a}(x)$ ) gauge field $B_{\mu}^{a}(x)$ with the mass $m_{B}$ (for details see $[8,13]$. CP is characterized by $k_{G L} \rightarrow \infty$ because of infinite fluctuation length $\xi \sim m_{B}^{-1}$. The second central moment (dispersion) $M_{2}$ of (3.2) will be divergent at $\lambda \rightarrow 1$, thus the fluctuation length (the effective radius of the flux tube) $\xi \sim \sqrt{\left|M_{2}\right|} \rightarrow \infty$. The finite values of $\xi$ will provide the analytical form of $G\left(k ; \lambda, \mu_{c}\right)$, however the very large fluctuation length gives the non-analytical behavior of $G\left(k ; \lambda, \mu_{c}\right)$ at $k \rightarrow 0$. The dual QCD vacuum (through $k_{G L}$ ) will infuence $\xi$ up to cross-over which is the unified process of phase transition between BM and AM.

In some sense, $G(k ; \lambda)$ can be understand in terms of CBE function (2.1) in the form

$$
G\left(k ; \lambda, \mu_{c}\right)=\frac{2-\bar{C}_{2}(0)}{2 \pi} \sum_{j=0}^{\infty}\left[\bar{C}_{2}(0)-1\right]^{j} \cos \left(\frac{k}{\mu_{c}} a^{j}\right)
$$

where $\bar{C}_{2}(0) \equiv \bar{C}_{2}(q=0 ; \lambda)=C_{2}(q=0 ; \lambda) / \eta(N)$ which can be extracted from an experiment. The divergence of (3.9) is expected if $\bar{C}_{2}(0) \rightarrow 2$ at high multiplicities $N$.

The characteristic feature of the model is the fluctuation length (3.4) and its analytical and non-analytical behavior at all possible $0<\lambda<1$ and $\mu_{c}$. The solution for BM can be considered in the form of Taylor's expansion [19]

$$
G_{B M}\left[k ; \lambda(S), \mu_{c}\right]=1+\sum_{s=1}^{S-1}(-1)^{s} \frac{1}{(2 s) !} \xi_{(2 s)}^{2}[\lambda(S)] k^{2 s},
$$

which is regular at $0<\lambda<1$ with finite $S(S \rightarrow \infty$ as $\lambda \rightarrow 0$ ). The rest term for AM is singular for $\xi_{(2 s)}^{2}$ at $s \geq S:$

$$
G_{A M}\left[k ; \lambda(S), \mu_{c}\right]=1+\sum_{s=S}^{\infty}(i)^{2 s} \frac{1}{(2 s) !} \xi_{(2 s)}^{2}[\lambda(S)] k^{2 s} .
$$

Actually, for some values of $\lambda(S)$ at given $S$ with $k \rightarrow 0$ the rest (singular) term (3.11) can give the main contribution to (3.5) and, thus, shall define the asymptotic behavior of $G\left(k ; \lambda, \mu_{c}\right)$ at $k \rightarrow 0$ and, consequently, the probability $P\left(x ; \lambda, \mu_{c}\right)$ in (3.2) at $x \rightarrow \infty$. The latter is in the correspondence with the infinite size of the particle source in terms of $\mathrm{CBE}$ at $\mathrm{CP}$. 
The special singular solution for AM may be presented in the $k$-power form [19]. Neglecting $\mathrm{BM}$ term (3.6) in (3.5) the infinite series (3.11) with the integer even powers $2 s$ of $k$ is replaced by

$$
G_{A M}\left[k ; \lambda(S), \mu_{c}\right]=C[\lambda(S)]\left|\frac{k}{\mu_{c}}\right|^{r[\lambda(S)]} Q(|k|),
$$

where $r$ is restricted in some interval of $S$ to be defined later. For any finite value $\lambda$ from an open interval $(0,1)$ there will be the finite value $S(\lambda)$ from the semi-open interval $[0,1)$, consequently, the inverse function $\lambda(S)$ does exist as well. The values of $\xi_{(2 s)}^{2}(\lambda)$ are finite for $0<\lambda<1$ if $s=1,2, \ldots, S-1$, and they will diverge if $s \geq S$. So, the series (3.10) is brought to the term $k^{2(S-1)}$.

Inserting (3.12) into the reduced homogenious equation (3.5) for AM, one finds

$$
r[\lambda(S)]=\frac{\ln \left[\frac{Q(|k|)}{Q(a|k|) \cdot \lambda(S)}\right]}{\ln a}, a>1
$$

Actually, $Q(a|k|)$ is the associated function of the 1 st order to the measure $\gamma$ with the proper function of the operator of dilatation transformation $u, u Q(|k|)=Q(a|k|)$. For any $a \geq 1$ the function $Q(a|k|)$ obeys the following condition [20]

$$
Q(a|k|)=a^{\gamma} Q(|k|)+a^{\gamma} \ln (a) \cdot Q_{0}(|k|),
$$

where $Q_{0}$ is the homogenious function of the measure $\gamma$. For $a$ close to unity (from above) one finds

$$
r[\lambda(S)]=-\left[\frac{\ln \lambda(S)}{\ln a}+\gamma\right]
$$

The singularity of (3.12) is supported by the inequality

$$
2(S+1)-\gamma \ln a \geq r(S)>2 S-\gamma \ln a .
$$

The coefficient $C[\lambda(S)]$ in (3.12) is

$$
C[\lambda(S)]=\frac{i^{r-2}}{\Gamma(r-1)} \xi_{(r-2)}^{2}(\lambda) .
$$

The solution for $Q(|k|)$ in (3.12) obeying the functional dilatation equation $u Q(|k|) \simeq Q(a \cdot|k|)$ contains the smoothly changing function which will be, e.g., the logarithmic-periodic function with the period $\log (a)>0$. If one replaces $|k| \rightarrow \log (|k|), a \cdot|k| \rightarrow \log (a)+\log (|k|)$, the solution of corresponding log-periodic equation is the infinite series

$$
Q(|k|) \sim \frac{1}{\log (a)} \sum_{m=0}^{\infty} b_{m} \cos \left[2 \pi m \frac{\log (|k|)}{\log (a)}+\varphi_{m}\right],
$$

where $b_{m}$ and $\varphi_{m}$ are coefficients and the phases, respectively, which are not important for the model considered here. Thus, the solution for AM looks like

$$
G_{A M}\left(k ; \lambda, \mu_{c}\right) \sim \frac{i^{r-2}}{\Gamma(r-1)} \xi_{(r-2)}^{2}(\lambda)\left|\frac{k}{\mu_{c}}\right|^{r} \frac{1}{\log (a)} \sum_{m=0}^{\infty} b_{m} \cos \left[2 \pi m \frac{\log (|k|)}{\log (a)}+\varphi_{m}\right],
$$


which is divergent if $\lambda \rightarrow 1$ (because of $\xi^{2}$ ), and $a \rightarrow 1$ from above (because of $\log (a)$ in the denominator of (3.13). The upper limit of the critical chemical potential is

$$
\mu_{c} \leq \mu\left[1+\frac{1}{n k_{G L}^{2}} O\left(\frac{m_{\phi}^{2}}{m^{2}}\right)\right]^{-1}
$$

and the cross-over $\left(\mu=\mu_{c}\right)$ does occur when $k_{G L} \rightarrow \infty$. The AM solution disappears if $r(S) / S \rightarrow 2$ as $S \rightarrow \infty$ at $\lambda(S) \rightarrow 0$.

\section{Conclusions}

The theoretical search for CP, in paricular, the cross-over between BM and AM, is studied in one-dimensional model of random fluctuation walk accompanied by the known model of CBE. The main points here are the random fluctuation weight $\bar{\lambda}$ in (3.1), the even moments of $G$ or the fluctuation length squared $\xi^{2}$, and the running parameter $a>1 . \bar{\lambda}$ is the stochastic random function $\lambda(v)$ which defines the strength of correlations between two identical particles with Bosestatistics. This depends very strongly of the vacuum properties characterized by Ginzburg-Landau parameter $k_{G L}$. It is shown that the solution is given in terms of the regular and the singular parts corresponding to BM and AM, respectively. The smooth $\lambda$ provides BM, however, if $\lambda \rightarrow 1$ (very strong influence of chaoticity) the asymptotic behavior at $k \rightarrow 0$ (or the probabiilty $P(x)$ for $x \rightarrow \infty$ ) is approached. The latter corresponds to infinite size $L_{s t}$ of a particle source .

\section{References}

[1] A.B. Migdal, Rev. Mod. Phys. 50 (1978) 107.

[2] D. Kharzeev, Phys. Lett. B633 (2006) 260.

[3] R. Arnaldi et al. [NA60 Collaboration], Phys. Rev. Lett. 96 (2006) 162302; A. Adare [PHENIX Collaboration], Phys. Rev. C 81 (2010) 034911.

[4] A.A. Andrianov et. al., Phys. Lett. B 710 (2012) 230.

[5] J. Zinn-Justin, Quantum Field Theory and Critical Phenomena, International Series of Monographs on Physics 77, Oxford Univ. Press, Oxford, 1989.

[6] H. Kleinert, V. Schulte-Frohlinde, Critical Properties of $\phi^{4}$-Theories, World Sci., Singapore, 2001.

[7] A.N. Vasiliev, Quantum Field RG in the Theory of Critical Behavior and Stochastic Dynamics, Sankt-Peterburg, PINP Publ. House, 1998.

[8] G.A. Kozlov, AIP Conf. Proc. 1701 (2016) 060013; Nucl. Part. Phys. Proc. 273-275 (2016) 2584.

[9] G.A. Kozlov, Phys. Part. Nucl. Lett. 6 (2009) 97.

[10] G.A. Kozlov, Phys. Rev. C58 (1998) 1188; J. Math. Phys. 42 (2001) 4749.

[11] CMS Collaboration, JHEP 05 (2011) 029.

[12] L3 Collaboration, Eur. Phys. J. C71 (2011) 1648.

[13] G.A. Kozlov, The critical end point through observables, arXiv:1503.05211 [hep-ph]. 
[14] OPAL Collaboration, Eur. Phys. J. C52 (2007) 787.

[15] CMS Collaboration, Phys. Rev. Lett. 105 (2010) 032001.

[16] ALICE Collaboration, Phys. Rev. D84 (2011) 112004.

[17] ATLAS Collaboration, Eur. Phys. J. C75 (2015) 466.

[18] C.-Y. Wong et al., Chaoticity and Coherence in Bose-Einstein Condensation and Correlations, talk at the Xth Workshop on Particle Correlations and Femtoscopy (WPCF14), Hungary, 2014; J. Central Eur. Green Innovations 4 (2016) 64.

[19] Yu. G. Rudoi, O.A. Kotelnikova, Theoretical and Mathematical Physics 189 (2016) 1818.

[20] I.M. Gelfand, G.E. Shilov, Generalized functions, Volume 1, Properties and Operators, AMS Chelsea Publishing (1964). 